\title{
Analysis of Influencing Factors on Survival Time of Patients with Heart Failure
}

\author{
Jianwei Sheng1, Xiyuan Qian1, Tong Ruan² \\ ${ }^{1}$ School of Science, East China University of Science and Technology, Shanghai, China \\ ${ }^{2}$ School of Information Science and Engineering, East China University of Science and Technology, Shanghai, China \\ Email: xyqian@ecust.edu.cn
}

How to cite this paper: Sheng, J.W., Qian, X.Y. and Ruan, T. (2018) Analysis of Influencing Factors on Survival Time of Patients with Heart Failure. Open Journal of Statistics, 8, 651-659. https://doi.org/10.4236/ojs.2018.84042

Received: May 6, 2018

Accepted: July 16, 2018

Published: July 19, 2018

Copyright ( 92018 by authors and Scientific Research Publishing Inc. This work is licensed under the Creative Commons Attribution International License (CC BY 4.0).

http://creativecommons.org/licenses/by/4.0/

(c) (i) Open Access

\begin{abstract}
To explore the influencing factors of survival time of patients with heart failure, a total of 1789 patients with heart failure were collected from Shanghai Shuguang Hospital. The Cox proportional hazards model and the mixed effects Cox model were used to analyze the factors on survival time of patients. The results of Cox proportional hazards model showed that age $(R R=1.32)$, hypertension $(\mathrm{RR}=0.67)$, ARB $(\mathrm{RR}=0.55)$, diuretic $(\mathrm{RR}=1.48)$ and antiplatelet $(R R=0.53)$ have significant impacts on the survival time of patients. The results of mixed effects Cox model showed that age $(R R=1.16)$, hypertension $(\mathrm{RR}=0.61)$, lung infection $(\mathrm{RR}=1.43)$, $\mathrm{ARB}(\mathrm{RR}=0.64), \beta$-blockers $(R R=0.77)$ and antiplatelet $(R R=0.69)$ have a significant impact on the survival time of patients. The results are consistent with the covariates age, hypertension, ARB and antiplatelet but inconsistent with the covariates lung infection and $\beta$-blockers.
\end{abstract}

\section{Keywords}

Heart Failure, Survival Analysis, Longitudinal Data, Mixed Effects Cox Model

\section{Introduction}

Heart failure is a syndrome with symptoms and signs caused by cardiac dysfunction, resulting in reduced longevity [1]. The prevalence of heart failure in western countries is $1 \%-2 \%$ of the adult population and $5-10$ per 1000 population per year, respectively [2] [3]. In China, the prevalence of heart failure in Chinese population aged $35-74$ is $0.9 \%$ and the population significantly increases with age [4] [5]. With the acceleration of population aging in China, it is foreseeable that the burden caused by heart failure will become heavier in the near future. So it is important to study and analyze the influencing factors of the survival time 
of patients with heart failure.

In medical research, follow-up is the common way to study the law of things; for instance: study the efficacy of a drug, study the survival time after surgery, study the lifetime of a medical device [6] [7]. The common ground of the above studies is that it will take some time to trace the research objects, which was called the survival time in statistics. The study of the distribution and influencing factors of survival time is the so-called survival analysis [8] [9] [10]. Proportional hazard regression model has become the most common used procedure for modeling the relationship of covariates to a survival or other censored outcome since this model was proposed by D.R. Cox in 1972 [11]. In clinical practice, many studies collect both longitudinal data [12] [13] (longitudinal data are data in which a response variable is measured at different time points over time) and survival-time data. In this paper, Cox proportional hazards model was used to model the survival-time data and mixed effects Cox model [14] [15] was used to model the survival-time and longitudinal data.

\section{Models}

\subsection{Cox Proportional Hazards Model}

The Cox proportional hazards model was proposed by British statistician D.R. Cox in 1972, which has been widely applied to analyze the effect of exposure and other covariates on patient's survival. The Cox model specifies the hazard for individual $i$ as:

$$
\lambda_{i}(t)=\lambda_{0}(t) \exp \left(\beta_{1} X_{i 1}+\beta_{2} X_{i 2}+\cdots+\beta_{p} X_{i p}\right)=\lambda_{0}(t) \exp \left(X_{i}(t) \beta\right)
$$

where $\beta=\left(\beta_{1}, \beta_{2}, \cdots \beta_{p}\right)^{\mathrm{T}}$ is a $p \times 1$ column vector of coefficients, $X_{i}=\left(X_{i 1}, X_{i 2}, \cdots, X_{i p}\right)$ is a $1 \times p$ vector of covariates for subject $i$, and $\lambda_{0}(t)$ is an unspecified nonnegative function of time called the baseline hazard, describing how the risk of event per time unit changes over time at baseline levels of covariates. Since the hazard ratio for two subjects with fixed covariate vectors $X_{i}$ and $X_{j}$

$$
\frac{\lambda_{i}(t)}{\lambda_{j}(t)}=\frac{\lambda_{0}(t) \exp \left(X_{i} \beta\right)}{\lambda_{0}(t) \exp \left(X_{j} \beta\right)}=\exp \left(\left(X_{i}-X_{j}\right) \beta\right)
$$

is constant over time, the model is called proportional hazards model.

Let the event be observed to have occurred with subject $i$ at time $t_{i}$. The probability that happened can be written as

$$
L_{i}(\beta)=\frac{\lambda\left(t_{i} \mid X_{i}\right)}{\sum_{: t_{j} \geq t_{i}} \lambda\left(t_{i} \mid X_{j}\right)}=\frac{\theta_{i}}{\sum_{: t_{j} \geq t_{i}} \theta_{j}}
$$

where $\theta_{j}=\exp \left(X_{j} \beta\right)$ and the summation is over the set of subjects $j$ who is still under observation at time $t_{i}$, the set is called risk set and denoted by $R\left(t_{i}\right)$, this is the partial likelihood for subject $i$. So taking the product of Equation (3) yields the partial likelihood function: 


$$
P L(\beta)=\prod_{i=1}^{n}\left[\frac{\exp \left(X_{i} \beta\right)}{\sum_{j \in R\left(t_{i}\right)} \exp \left(X_{j} \beta\right)}\right]^{\delta_{i}}
$$

where $\delta_{i}$ is 1 if the event is happened to subject $i$ and 0 otherwise.

\subsection{Mixed Effects Cox Model}

In clinical practice, some subjects may be observed more than once during the time from first hospitalization to death. The number of hospitalizations and the days between two hospitalizations varies from patient to patient in the heart failure set. The Cox proportional hazards model only uses the survival-time data, which inevitably lose some useful information. The data obtained from multiple measurements of a series of experimental individuals over time are called longitudinal data. More precisely, suppose there are $m$ individuals in an experiment where each individual is measured over time. $Y_{i 1}, Y_{i 2}, \cdots Y_{i n_{i}}, i=1, \cdots, m$ are the measured data for the individual $i$ at time $t_{i 1}<t_{i 2}<\cdots<t_{i n_{i}}$, then $\left\{Y_{i k}: 1 \leq k \leq n_{i}, 1 \leq i \leq m\right\}$ is called longitudinal data, which is also called panel data in econometrics [16]. This type of data is different from cross-section data and time series data. The linear mixed effects model is a common model to dealing with the longitudinal data [17]. It adds individual difference as random effects into the regression model. These random effects describe how every object's measurement changes over time and reflect the internal structure of the longitudinal data. In matrix notation a mixed model can be represented as:

$$
Y=X^{\mathrm{T}} \beta+Z^{\mathrm{T}} b+\varepsilon, b \sim N(0, \Sigma)
$$

where $\mathrm{X}$ and $\mathrm{Z}$ are the design matrices for the fixed and random effects respectively, $\beta$ is the vector of fixed-effects coefficients and $b$ is the vector of random effects coefficients and $\varepsilon$ is the random error. The random effects distribution is modeled as Gaussian with mean zero and a variance matrix $\Sigma$. Combining Equation (1) and (3) yields the mixed effects Cox model:

$$
\lambda(t)=\lambda_{0}(t) \exp \left(X^{\mathrm{T}} \beta+Z^{\mathrm{T}} b\right), b \sim N(0, \Sigma)
$$

Coefficients can be estimated based on the partial likelihood:

$$
\ln [P L(\beta, b)]=\sum_{i=1}^{n} \int_{0}^{\infty}\left\{Y_{i}(t) \eta_{i}(t)-\ln \left[\sum_{j} Y_{j}(t) \exp \left(\eta_{j}(t)\right)\right]\right\} \mathrm{d} t
$$

where $\eta_{i}(t)=X_{i}(t) \beta+Z_{i}(t) b$ is the linear score for subject $i$ at time $t$ and $Y_{i}(t)=1$ if subject $i$ is still under observation at time $t$ and 0 otherwise [18] [19].

\section{Data}

We collected patient basic information, laboratory information, medical records, doctor's advice information and other information from Shanghai Shuguang Hospital database during January 1, 2003 to December 31, 2013. The start point of survival analysis is the first time in hospital date and the end point is the last 
time out of hospital date or the date of death or the end date of the study. According to the guidance of the doctor formed the heart failure dataset used in this paper. This dataset contains data from 1789 patients with heart failure, for a total of 8332 observations and 23 covariates. See Table 1 for details.

Most are categorical variables, but age is a multi-variable. Its distribution is shown in Figure 1.

Statistics for other binary variables are shown in Table 2.

\section{Results}

Firstly, we use the Cox proportional hazards to model the survival-time data with all covariates. The results are shown in Table 3.

Table 1. Variables description in heart failure dataset.

\begin{tabular}{|c|c|c|}
\hline variables & Description & Data Type \\
\hline Id & Patient id & Categorical \\
\hline num & Hospitalization number of patients & Categorical \\
\hline Status & $1=$ dead, $0=$ alive & Binary \\
\hline day & $\begin{array}{l}\text { Number of days between first hospitalization and } \\
\text { death or last out of hospital }\end{array}$ & Numeric \\
\hline days & $\begin{array}{c}\text { Number of days between first hospitalization and } \\
\text { this in hospitalization date }\end{array}$ & Numeric \\
\hline age & $\begin{array}{c}1=(0,40], 2=(41,50], 3=(51,60], 4=(61,70], \\
5=(71,80], 6=(81,90], 7=(91,100]\end{array}$ & Multi-category \\
\hline sex & $1=$ male, $0=$ female & Binary \\
\hline Chin_Med & Whether used Chinese Medicine? $1=$ yes, $0=$ no & Binary \\
\hline $\mathrm{RBC}$ & Red blood cells in $\mathrm{mg} / \mathrm{ml}$ & Numeric \\
\hline HGB & Hemoglobin in $\mathrm{mg} / \mathrm{ml}$ & Numeric \\
\hline hypertension & Presence of hypertension, $1=$ yes, $0=$ no & Binary \\
\hline coronary & Presence of coronary heart disease, $1=$ yes, $0=$ no & Binary \\
\hline diabetes & Presence of diabetes, $1=$ yes, $0=$ no & Binary \\
\hline lung_infe & Presence of lung infection, $1=$ yes, $0=$ no & Binary \\
\hline bronchitis & Presence of chronic bronchitis, $1=$ yes, $0=$ no & Binary \\
\hline ACEI & $\begin{array}{l}\text { Whether used angiotensin converting enzyme } \\
\text { inhibitors? } 1=\text { yes, } 0=\text { no }\end{array}$ & Binary \\
\hline ARA & $\begin{array}{l}\text { Whether used aldosterone receptor antagonists? } \\
\qquad 1=\text { yes, } 0=\text { no }\end{array}$ & Binary \\
\hline $\mathrm{ARB}$ & $\begin{array}{l}\text { Whether used angiotensin receptor blocker? } \\
\qquad 1=\text { yes, } 0=\text { no }\end{array}$ & Binary \\
\hline Blocker & Whether used $\beta$ blocker? $1=$ yes, $0=$ no & Binary \\
\hline diuretic & Whether used Diuretic? $1=$ yes, $0=$ no & Binary \\
\hline digitalis & Whether used digitalis? $1=$ yes, $0=$ no & Binary \\
\hline anti-platelet & Whether used anti-platelet? $1=$ yes, $0=$ no & Binary \\
\hline nitrate & Whether used nitrate? $1=$ yes, $0=$ no & Binary \\
\hline
\end{tabular}


Table 2. Statistics for binary variable in hear failure set (total = 1789).

\begin{tabular}{|c|c|c|}
\hline variables & & N (\%) \\
\hline \multirow{2}{*}{ status } & alive & $1531(85.6)$ \\
\hline & death & $258(14.4)$ \\
\hline \multirow{2}{*}{$\operatorname{sex}$} & male & $955(53.3)$ \\
\hline & female & $834(46.7)$ \\
\hline \multirow{2}{*}{ chin_med } & yes & $1337(74.7)$ \\
\hline & no & $834(25.3)$ \\
\hline \multirow{2}{*}{ coronary } & yes & $501(28)$ \\
\hline & no & $1288(72)$ \\
\hline \multirow{2}{*}{ hypertension } & yes & $1119(62.6)$ \\
\hline & no & $670(37.4)$ \\
\hline \multirow{2}{*}{ diabetes } & yes & $498(27.8)$ \\
\hline & no & $1291(72.2)$ \\
\hline \multirow{2}{*}{ lung_infe } & yes & $215(12)$ \\
\hline & no & $1574(88)$ \\
\hline \multirow{2}{*}{ bronchitis } & yes & $246(13.7)$ \\
\hline & no & $1543(86.3)$ \\
\hline \multirow{2}{*}{ ACEI } & yes & 392 (21.9) \\
\hline & no & $1937(78.1)$ \\
\hline \multirow{2}{*}{ ARA } & yes & $373(20.8)$ \\
\hline & no & $1416(79.2)$ \\
\hline \multirow{2}{*}{$\mathrm{ARB}$} & yes & $361(20.2)$ \\
\hline & no & $1428(79.8)$ \\
\hline \multirow{2}{*}{ Blocker } & yes & $800(44.7)$ \\
\hline & no & $989(55.3)$ \\
\hline \multirow{2}{*}{ diuretic } & yes & $383(21.4)$ \\
\hline & no & $1406(78.6)$ \\
\hline \multirow{2}{*}{ digitalis } & yes & $1117(62.4)$ \\
\hline & no & $672(37.6)$ \\
\hline \multirow{2}{*}{ anti-platelet } & yes & 709 (39.6) \\
\hline & no & $1080(60.4)$ \\
\hline \multirow{2}{*}{ nitrate } & yes & $892(49.9)$ \\
\hline & no & $897(50.1)$ \\
\hline
\end{tabular}

Secondly, we use the mixed effects Cox model to model the survival-time data and longitudinal data with all the covariates and variable day as the covariate for random effects. The results are shown in Table 4.

\section{Conclusions}

Cox proportional hazards model showed that age, hypertension, ARB, diuretics and antiplatelet have a statistically significant effect on the survival time of patients. Age $(R R=1.32)$ and diuretic $(R R=1.48)$ were risk factors. Hypertension $(\mathrm{RR}=0.67), \mathrm{ARB}(\mathrm{RR}=0.55)$ and antiplatelet $(\mathrm{RR}=0.53)$ were protective factors. The mixed effects Cox model showed that age, hypertension, lung infection, $\mathrm{ARB}, \beta$-blockers, and antiplatelet have statistically significant effects on the survival time of patients. Age $(R R=1.16)$ and lung infection $(R R=1.43)$ were risk 
distribution of patients age

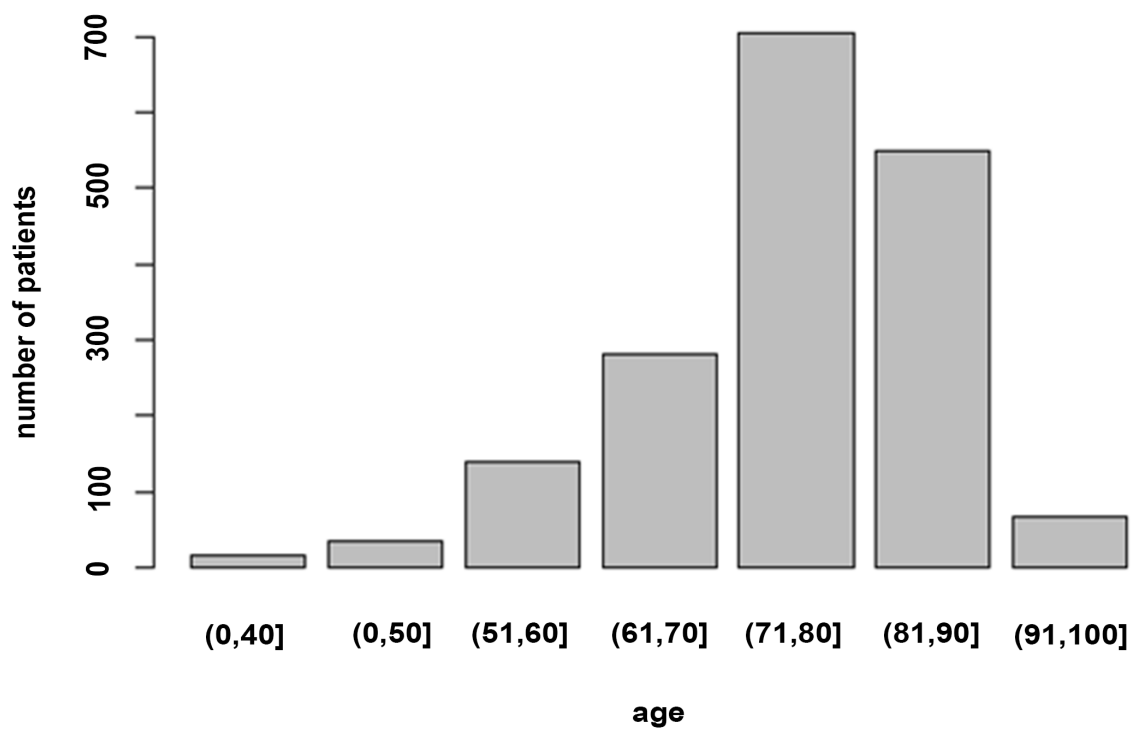

Figure 1. Distribution of heart failure patients' age.

Table 3. Result of Cox proportional hazards model with all covariates.

\begin{tabular}{cccccc}
\hline variables & coef & RR & Se (coef) & $\mathrm{z}$ & p-value \\
\hline sex & 0.222649 & 1.249383 & 0.178811 & 1.245 & 0.21307 \\
age & 0.275551 & 1.317256 & 0.097916 & 2.814 & 0.00489 \\
Chin_med & -0.31796 & 0.727633 & 0.200295 & -1.587 & 0.11241 \\
RBC & -0.1807 & 0.834684 & 0.244466 & -0.739 & 0.4598 \\
HGB & -0.00859 & 0.991447 & 0.007816 & -1.099 & 0.27175 \\
hypertension & -0.40512 & 0.6669 & 0.196386 & -2.063 & 0.03913 \\
coronary & 0.029494 & 1.029934 & 0.203818 & 0.145 & 0.88494 \\
diabetes & -0.01215 & 0.987926 & 0.22145 & -0.055 & 0.95625 \\
lung_infe & -0.26373 & 0.768185 & 0.307327 & -0.858 & 0.39082 \\
bronchitis & 0.218949 & 1.244768 & 0.21796 & 1.005 & 0.31512 \\
ACEI & -0.24764 & 0.780638 & 0.240374 & -1.03 & 0.3029 \\
ARA & -0.27402 & 0.760313 & 0.21431 & -1.279 & 0.20102 \\
ARB & -0.60086 & 0.54834 & 0.266228 & -2.257 & 0.02401 \\
Bblocker & -0.19269 & 0.824737 & 0.186844 & -1.031 & 0.3024 \\
diuretic & 0.389164 & 1.475747 & 0.191756 & 2.029 & 0.04241 \\
digitalis & 0.305065 & 1.356714 & 0.184673 & 1.652 & 0.09855 \\
anti-platelet & -0.64137 & 0.526573 & 0.206546 & -3.105 & 0.0019 \\
nitrate & 0.319543 & 1.376498 & 0.173849 & 1.838 & 0.06605 \\
\hline
\end{tabular}

${ }^{*}$ coef is the estimation of the coefficients; RR is relative risk; Se (coef) is the standard error of the estimation. 
Table 4. Results of mixed effects Cox model.

\begin{tabular}{|c|c|c|c|c|c|}
\hline variables & coef & RR & Se (coef) & $\mathrm{z}$ & p-value \\
\hline sex & 0.301405 & 1.351757 & 0.161594 & 1.87 & 0.062 \\
\hline age & 0.144165 & 1.155074 & 0.067629 & 2.13 & 0.033 \\
\hline Chin_med & -0.02249 & 0.977757 & 0.082878 & -0.27 & 0.79 \\
\hline $\mathrm{RBC}$ & -0.1126 & 0.893511 & 0.169517 & -0.66 & 0.51 \\
\hline HGB & -0.01085 & 0.989209 & 0.005333 & -2.03 & 0.042 \\
\hline hypertension & -0.49125 & 0.611863 & 0.127701 & -3.85 & 0.00012 \\
\hline coronary & -0.1687 & 0.844765 & 0.140132 & -1.2 & 0.23 \\
\hline diabetes & -0.23967 & 0.786885 & 0.161708 & -1.48 & 0.14 \\
\hline lung_infe & 0.356836 & 1.428802 & 0.124253 & 2.87 & 0.0041 \\
\hline bronchitis & 0.250458 & 1.284613 & 0.148653 & 1.68 & 0.092 \\
\hline ACEI & -0.32509 & 0.722463 & 0.154382 & -2.11 & 0.035 \\
\hline ARA & 0.069231 & 1.071684 & 0.123429 & 0.56 & 0.57 \\
\hline ARB & -0.44209 & 0.642691 & 0.122451 & -3.61 & 0.00031 \\
\hline Bblocker & -0.26293 & 0.768796 & 0.089191 & -2.95 & 0.0032 \\
\hline diuretic & 0.115389 & 1.12231 & 0.104295 & 1.11 & 0.27 \\
\hline digitalis & 0.037052 & 1.037747 & 0.081806 & 0.45 & 0.65 \\
\hline anti-platelet & -0.3711 & 0.689975 & 0.101789 & -3.65 & 0.00027 \\
\hline nitrate & 0.029271 & 1.029703 & 0.086633 & 0.34 & 0.74 \\
\hline
\end{tabular}

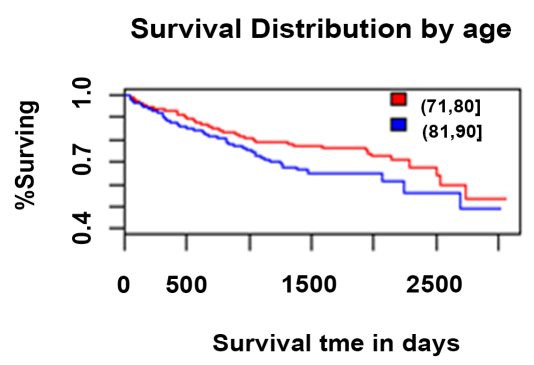

Survival Distribution by hypertension

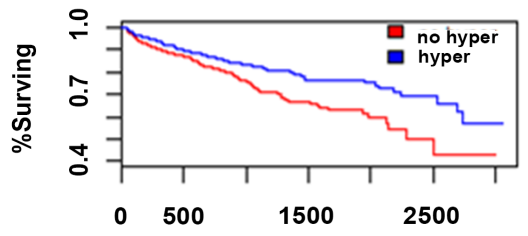

Survival tme in days

\section{Survival Distribution by ARB}

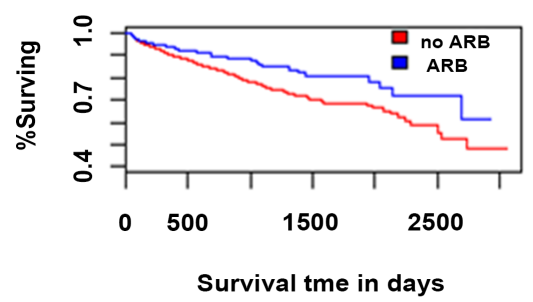

Survival Distribution by anti-platelet

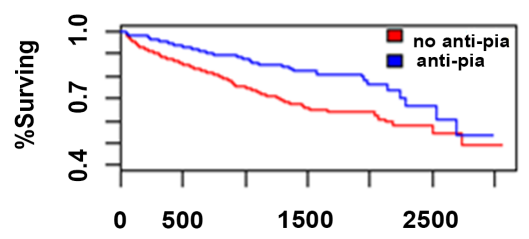

Survival tme in days

Figure 2. Survival distributions by significant covariates.

factors; hypertension $(\mathrm{RR}=0.61)$, ARB $(\mathrm{RR}=0.64), \beta$ blockers $(\mathrm{RR}=0.77)$ and antiplatelet $(\mathrm{RR}=0.69)$ were protective factors. Results of the two models are 
consistent with the covariates age, hypertension, ARB and antiplatelet. Further, age was risk factor, namely the older has lower survival rate. Hypertension, ARB, and antiplatelet were protective factors, namely patients with hypertension have higher survival rates than those without hypertension; patients who used ARBs had higher survival rates than unused patients; patients who used antiplatelet drugs had higher survival rates than those who did not. Survival distributions by these covariates are shown in Figure 2.

The difference is that there are another two covariates which have significantly effect on the survival rate in the mixed effects Cox model: one was risk factor lung infection $(\mathrm{RR}=1.43)$, and the other was protective factor $\beta$ blocker $(\mathrm{RR}=$ 0.67). In addition, the protective factor diuretic in the Cox proportional hazards model became insignificant in the mixed effects Cox model, which shows that the effect of diuretics on survival rate gradually reduces.

\section{Acknowledgements}

This work was partially supported by The National High-Tech R\&D Program of China (863 Program) under Grant No. 2015AA020107.

\section{References}

[1] Mosterd, A. and Hoes, A.W. (2007) Clinical Epidemiology of Heart Failure. Heart, 93, 1137-1146. https://doi.org/10.1136/hrt.2003.025270

[2] McMurray, J.J., Adamopoulos, S., Anker, S.D., et al. (2012) ESC Guidelines for the Diagnosis and Treatment of Acute and Chronic Heart Failure. European Heart Journal, 33, 1787-1146.

[3] Cowie, M.R., Wood, D.A., Coats, A.J., et al. (1999) Incidence an Aetiology of Heart Failure: A Population-Based Study. European Heart Journal, 20, 421-428. https://doi.org/10.1053/euhj.1998.1280

[4] Guo, Y., Zhao, D. and Liu, J. (2015) Epidemiological Study of Heart Failure in China. Cardiovascular Innovations and Applications, 1, 47-55. https://doi.org/10.15212/CVIA.2015.0003

[5] Gu, D.F., Huang, G.Y., He, J., et al. (2003) Investigation of Prevalence and Distribution Feature of Chronic Heart Failure in Chinese Adult Population. Chinese Journal Cardio, 1, 3-6.

[6] Bland, J.M. (2000) An Introduction to Medical Statistics. 3rd Edition, Oxford University Press, New York.

[7] Kirkwood, B.R. and Sterne, J.C. (2003) Essential Medical Statistics. 2nd Edition, Blackwell Publishers, Malden.

[8] Klein, J.P. (2013) Handbook of Survival Analysis. CRC Press, Boca Raton.

[9] Lawless, J.F. (2003) Statistical Models and Methods for Lifetime Data. 2nd Edition, John Wiley and Sons, New York.

[10] Kalbfleisch, J.D. and Prentice, R.L. (2002) The Statistical Analysis of Failure Time Data. John Wiley and Sons, New York. https://doi.org/10.1002/9781118032985

[11] Cox, D.R. (1972) Regression Models and Lifetables (with Discussion). Journal of the Royal Statistical Society B, 34, 187-220.

[12] Frees, E. (2004) Longitudinal and Panel Data: Analysis and Applications in the So- 
cial Sciences. Cambridge University Press, New York. https://doi.org/10.1017/CBO9780511790928

[13] Diggle, P.J., Heagerty, P., Liang, K.-Y. and Zeger, S.L. (2002) Analysis of Longitudinal Data. 2nd Edition, Oxford University Press, Oxford.

[14] Therneau. T.M. (2015) Mixed Effects Cox Models. BMC Genetics, 6, S127.

[15] Therneau, T.M. and Grambsch, P.M. (2000) Modeling Survival Data: Extending the Cox Model. Springer, New York. https://doi.org/10.1007/978-1-4757-3294-8

[16] Wooldridge, J.M. (2013) Introductory Econometrics: A Modern Approach. 5th Edition, South-Western, Mason, OH.

[17] Militino, A.F. (2010) Mixed Effects Models and Extensions in Ecology with R. Journal of Royal Statistical Society, 173, 938-939.

https://doi.org/10.1111/j.1467-985X.2010.00663_9.x

[18] Ripatti, S. and Palmgren, J. (2000) Estimation of Multivariate Frailty Models Using Penalized Partial Likelihood. Biometrics, 56, 1016-1022. https://doi.org/10.1111/j.0006-341X.2000.01016.x

[19] Gamst, A., Donohue, M. and Xu, R. (2009) Asymptotic Properties and Empirical Evaluation of the Npmle in the Proportional Hazards Mixed-Effects Model. Statistical Sinica, 19, 997-1011. 